\title{
On two-parametric family of quartic Thue equations
}

\author{
par BORKA JADRIJEVIĆ
}

RÉSUMÉ. Nous montrons que pour tous les entiers $m$ et $n$, il n'y a pas de solution non triviale de l'équation de Thue

$$
x^{4}-2 m n x^{3} y+2\left(m^{2}-n^{2}+1\right) x^{2} y^{2}+2 m n x y^{3}+y^{4}=1,
$$

satisfaisant la condition supplémentaire $\operatorname{pgcd}(x y, m n)=1$.

ABSTRACT. We show that for all integers $m$ and $n$ there are no non-trivial solutions of Thue equation

$$
x^{4}-2 m n x^{3} y+2\left(m^{2}-n^{2}+1\right) x^{2} y^{2}+2 m n x y^{3}+y^{4}=1,
$$

satisfying the additional condition $\operatorname{gcd}(x y, m n)=1$.

\section{Introduction}

One of the most famous Diophantine equation is equation

$$
F(x, y)=t
$$

where $F \in \mathbb{Z}[X, Y]$ is a homogeneous irreducible polynomial of degree $\geq 3$ and $t \neq 0$ a fixed integer. Equation (1.1) is called a Thue equation in honour of A. Thue, who proved in 1909 [20], that equation (1.1) has only finitely many integral solutions $(x, y)$. Thue's proof was non-effective. In 1968, Baker [1] showed, using estimates for linear forms in logarithms of algebraic numbers, that Thue equation can be solved effectively. The result of Baker implies that all solution of equation (1.1) can be found in finitely many steps, at least by direct enumeration. Baker's work were improved and generalized by many authors and general powerful methods have been developed for the explicit solution of Thue equations (see [5, 17, 23]), following from Baker's work. In 1990, Thomas [19] investigated for the first time a parametrized family of Thue equations. Since then, several families have been studied (see [10] for references). In particular, quartic families have been considered in $[6,7,10,11,12,13,15,16,18,21,24,25]$.

In [12], we considered the two-parametric family of quartic Thue equations

$$
x^{4}-2 m n x^{3} y+2\left(m^{2}-n^{2}+1\right) x^{2} y^{2}+2 m n x y^{3}+y^{4}=1,
$$


where parameters $m$ and $n$ are integers. Using the method of Tzanakis, given in [22], it was shown that solving equation (1.2) reduces to solving the system of Pellian equations

$$
\begin{aligned}
V^{2}-\left(m^{2}+2\right) U^{2} & =-2, \\
Z^{2}-\left(n^{2}-2\right) U^{2} & =2,
\end{aligned}
$$

for $n \neq 0, \pm 1$. The main result obtained in [12] can be stated as follows: If $|m|$ and $|n|$ are sufficiently large and have sufficiently large common divisor, then the system has only the trivial solutions $(V, Z, U)=( \pm m, \pm n, \pm 1)$, which implies that the original Thue equation also has only the trivial solutions $(x, y)=( \pm 1,0),(0, \pm 1)$. In [12], it was also shown that system (1.3) and (1.4) for all $m \geq 0$ and $n \geq 2$ possesses at most 7 solutions in positive integers $(V, Z, U)$.

The main goal of the present paper is to show that Thue equation (1.2) can be solved for all integers $m$ and $n$ if we impose the additional condition $\operatorname{gcd}(x y, m n)=1$ for the solutions $(x, y)$. This condition arises from the analyse of the connection between equation (1.2) and system (1.3) and (1.4). This leads us to consider three special cases: $m=n, m=2 n, n=2 m$. These cases are completely solved by applying a theorem of Bennett [4, Theorem 3.2] on simultaneous approximations of algebraic numbers. In all cases we obtain only trivial solutions, except for $m=1, n=2$. The case $m=2 n$ can be considered as a special case of the Thue equation

$$
x^{4}-4 c x^{3} y+(6 c+2) x^{2} y^{2}+4 c x y^{3}+y^{4}=1,
$$

which was completely solved in [7].

The main result of present paper is the following theorem.

Theorem 1.1. There are no solutions of (1.2) satisfying the additional conditions $\operatorname{gcd}(x y, m n)=1$ and $x y \neq 0$.

Let us note that, because of homogeneity and symmetry of equation (1.2), it is enough to consider the cases when $m$ and $n$ are nonnegative and find only all positive solutions. More precisely, $(x, y)=(a, b)$ is a solution of equation (1.2) then $(x, y)=(-a,-b),(b,-a),(-b, a)$ are solutions too. Thus, we will suppose, without loss of generality, that $m \geq 0$ and $n \geq 0$ are integers and consider equation of the form (1.2).

\section{The system of Pellian equations}

We will apply the method of Tzanakis introduced in [22] and used in $[7,8,12]$. Tzanakis showed that solving quartic Thue equations of the form

$$
f(x, y)=t
$$

$$
f(x, y)=a_{0} x^{4}+4 a_{1} x^{3} y+6 a_{2} x^{2} y^{2}+4 a_{3} x y^{3}+a_{4} y^{4} \in \mathbb{Z}[x, y], a_{0}>0,
$$


whose corresponding quartic field $\mathbf{K}$ is totally real, Galois and non-cyclic, reduces to solving a system of Pellian equations having one common unknown.

If we apply this method to the quartic Thue equation (1.2) we find (see [12, Section 4]) that if $n \geq 2$, then solving (1.2) reduces to solving the system of Pellian equations (1.3) and (1.4) with one common unknown, where

$$
\begin{aligned}
& U=\frac{G_{1}}{2}=x^{2}+y^{2}, \\
& V=\frac{G_{2}}{2}=m x^{2}+2 n x y-m y^{2}, \\
& Z=\frac{G_{3}}{2}=-n x^{2}+2 m x y+n y^{2} .
\end{aligned}
$$

All solutions of equation (1.3) for $m>0$ in positive integers are given by

$$
v+u \sqrt{m^{2}+2}=\left(m+\sqrt{m^{2}+2}\right)\left(m^{2}+1+m \sqrt{m^{2}+2}\right)^{k},
$$

where $k \in \mathbb{Z}$ and $k \geq 0$ or by $u=U_{k}$ and $v=V_{k}$, where the sequences $\left(U_{k}\right)$ and $\left(V_{k}\right)$ are defined by the recurrences

$$
\begin{aligned}
& U_{0}=1, \quad U_{1}=2 m^{2}+1, \quad U_{k+2}=2\left(m^{2}+1\right) U_{k+1}-U_{k}, k \geq 0 \\
& V_{0}=m, \quad V_{1}=m\left(2 m^{2}+3\right), \quad V_{k+2}=2\left(m^{2}+1\right) V_{k+1}-V_{k}, k \geq 0
\end{aligned}
$$

All solutions of equation (1.4) in positive integers are given by

$$
z+t \sqrt{n^{2}-2}=\left(n+\sqrt{n^{2}-2}\right)\left(n^{2}-1+n \sqrt{n^{2}-2}\right)^{l}
$$

where $l \in \mathbb{Z}$ and $l \geq 0$ or by $t=T_{l}$ and $z=Z_{l}$, where the sequences $\left(T_{l}\right)$ and $\left(Z_{l}\right)$ are defined by the recurrences

$$
\begin{array}{lll}
T_{0}=1, & T_{1}=2 n^{2}-1, & T_{l+2}=2\left(n^{2}-1\right) T_{l+1}-T_{l}, l \geq 0 \\
Z_{0}=n, & Z_{1}=n\left(2 n^{2}-3\right), & Z_{l+2}=2\left(n^{2}-1\right) Z_{l+1}-Z_{l}, l \geq 0 .
\end{array}
$$

In this way we reformulated the system of Pellian equations (1.3) and (1.4) to the Diophantine equation of the form

$$
U_{k}=T_{l}
$$

in integers $k, l \geq 0$. In order to prove that for some parameters $m>0$ and $n \geq 2$ we have only trivial solutions of (1.2), it suffices to show that $U_{k}=T_{l}$ implies $k=l=0$. 


\section{Some special cases}

Equation (1.2) has only trivial solutions for $n=0,1$ (see [12, Proposition $1, \mathrm{i})])$. For $m=0$, we have

$$
x^{4}+2\left(1-n^{2}\right) x^{2} y^{2}+y^{4}=1 .
$$

If the sequence $\left(n_{k}\right)$ is defined by

$$
n_{0}=1, \quad n_{1}=3, \quad n_{k+2}=6 n_{k+1}-n_{k}, \quad k \geq 0,
$$

then, for $n=n_{k}$ and $k \geq 1$, all non-trivial solutions of equation (3.1) are given by

$$
(x, y)=\left( \pm 1, \pm \sqrt{2\left(n^{2}-1\right)}\right) \text { and }\left( \pm \sqrt{2\left(n^{2}-1\right)}, \pm 1\right) .
$$

For all other values of $n$ we have only the trivial solutions $(x, y)=( \pm 1,0)$, $(0, \pm 1)$ by [12, Proposition 1, ii)] (see also [14] and [26]).

Now, consider three special cases: $m=n, m=2 n, n=2 m$.

Case 1. $m=n$.

If $m=n=c \geq 1$, then (1.2) has the form

$$
x^{4}-2 c^{2} x^{3} y+2 x^{2} y^{2}+2 c^{2} x y^{3}+y^{4}=1 .
$$

For $c \geq 2$, then solving (1.2) reduces to solving the system of Pellian equations

$$
\begin{aligned}
& c^{2} V_{1}^{2}-\left(c^{2}+2\right) U^{2}=-2, \\
& c^{2} Z_{1}^{2}-\left(c^{2}-2\right) U^{2}=2,
\end{aligned}
$$

where

$$
\begin{aligned}
U & =x^{2}+y^{2}, \\
V_{1} & =\frac{1}{c} V=x^{2}+2 x y-y^{2}, \\
Z_{1} & =\frac{1}{c} Z=-x^{2}+2 x y+y^{2} .
\end{aligned}
$$

Case 2. $m=2 n$.

If $m=2 c, n=c \geq 1$, then (1.2) has the form

$$
x^{4}-4 c^{2} x^{3} y+2\left(3 c^{2}+1\right) x^{2} y^{2}+4 c^{2} x y^{3}+y^{4}=1 .
$$

For $c \geq 2$, then solving (1.2) reduces to solving the system of Pellian equations

$$
\begin{gathered}
2 c^{2} V_{2}^{2}-\left(2 c^{2}+1\right) U^{2}=-1 \\
c^{2} Z_{2}^{2}-\left(c^{2}-2\right) U^{2}=2
\end{gathered}
$$


where

$$
\begin{aligned}
U & =x^{2}+y^{2}, \\
V_{2} & =\frac{1}{2 c} V=x^{2}+x y-y^{2}, \\
Z_{2} & =\frac{1}{c} Z=-x^{2}+4 x y+y^{2} .
\end{aligned}
$$

Case 3. $n=2 m$.

If $n=2 c, m=c \geq 1$, then (1.2) has the form

$$
x^{4}-4 c^{2} x^{3} y+2\left(1-3 c^{2}\right) x^{2} y^{2}+4 c^{2} x y^{3}+y^{4}=1 \text {. }
$$

For $c \geq 1$, then solving (1.2) reduces to solving the system of Pellian equations

$$
\begin{aligned}
c^{2} V_{3}^{2}-\left(c^{2}+2\right) U^{2} & =-2, \\
2 c^{2} Z_{3}^{2}-\left(2 c^{2}-1\right) U^{2} & =1
\end{aligned}
$$

where

$$
\begin{aligned}
U & =x^{2}+y^{2}, \\
V_{3} & =\frac{1}{c} V=x^{2}+4 x y-y^{2}, \\
Z_{3} & =\frac{1}{2 c} Z=-x^{2}+x y+y^{2} .
\end{aligned}
$$

Equations (3.3), (3.4), (3.5) we solve, in the same manner, as equation (1.5) in [7]. We find a lower bound for the solutions of the corresponding system of Pellian equations using the "congruence method" introduced in [9]. By comparison of this lower bound with an upper bound obtained from a theorem of Bennett [4] on simultaneous approximations of algebraic numbers we find only trivial solutions for $c \geq c_{0}$, where $c_{0}=235$ if $m=$ $n=c \geq 2, c_{0}=423$ if $m=2 c$ and $n=c \geq 2$ or $m=2 c$ and $n=c \geq 2$. For $c \leq c_{0}$ we use a theorem a Baker and Wüstholz [3] and a version of the reduction procedure due to Baker and Davenport [2]. In all cases we obtain only trivial solutions, except for $m=1, n=2$ where there are also non-trivial solutions $(x, y)=(4,5),(-4,-5),(5,-4),(-5,4)$.

Therefore, we have

Proposition 3.1. Equation (1.2) has only the trivial solutions $(x, y)=$ $( \pm 1,0),(0, \pm 1)$ in the following cases:

i) $n \leq 1$;

ii) $m=0$ and $2\left(n^{2}-1\right)$ is not a perfect square;

iii) $m=n \geq 2$;

iv) $m=2 n \geq 2$;

v) $n=2 m>2$; 
For cases for which there are also non-trivial solutions of equation (1.2) we have

Proposition 3.2. $\quad$ i) If $m=1$ and $n=2$ then all non-trivial solutions are $(x, y)=(4,5),(-4,-5),(5,-4),(-5,4)$;

ii) If $m=0$ and $2\left(n^{2}-1\right)$ is a perfect square then all non-trivial solutions are $(x, y)=\left( \pm 1, \pm \sqrt{2\left(n^{2}-1\right)}\right)$ and $\left( \pm \sqrt{2\left(n^{2}-1\right)}, \pm 1\right)$.

\section{Proof of the Theorem 1}

All solutions of the Pellian equation (1.3) in positive integers for $m>0$ are given by $(U, V)=\left(U_{k}, V_{k}\right)$, where the sequences $\left(U_{k}\right)$ and $\left(V_{k}\right)$ are defined by the recurrences (2.4) and (2.5). All solutions of the Pellian equation (1.4) in positive integers for $n \geq 2$ are given by $(U, Z)=\left(T_{l}, Z_{l}\right)$, where the sequences $\left(T_{l}\right)$ and $\left(Z_{l}\right)$ are defined by the recurrences (2.6) and (2.7). Recurrences (2.5) and (2.7) imply $m \mid V$ and $n \mid Z$.

Then, from (2.2) and (2.3), we have $m \mid 2 n x y$ and $n \mid 2 m x y$. If $\operatorname{gcd}(m, x y)$ $=1$ and $\operatorname{gcd}(n, x y)=1$, then $m \mid 2 n$ and $n \mid 2 m$, which implies $m=n$ or $m=2 n$ or $n=2 m$. In all these cases we obtain only trivial solutions, except for $m=1, n=2$. If $m=1$ and $n=2$, then, for non-trivial solutions $(x, y)$ we have $\operatorname{gcd}(n, x y)=\operatorname{gcd}(2, \pm 20)=2$.

It remains to consider the cases when $m=0$ and $n \leq 1$. For $n \leq 1$ equation (1.2) has only the trivial solutions. If $m=0$ then for some values of $n$ there are also non-trivial solutions of equation (1.2)(see Proposition 3.1 , ii) and Proposition 3.2, ii)). For those non-trivial solutions we have $\operatorname{gcd}(m, x y)=\operatorname{gcd}\left(0, \pm \sqrt{2\left(n^{2}-1\right)}\right)=\sqrt{2\left(n^{2}-1\right)}>2$. Therefore, we have proved Theorem 1 .

Acknowledgements: The author would like to thank Professor Andrej Dujella for helpful suggestions.

\section{References}

[1] A. BAKER, Contributions to the theory of Diophantine equations I. On the representation of integers by binary forms. Philos. Trans. Roy. Soc. London Ser. A 263 (1968), 173-191.

[2] A. BAKER, H. DAVENPORT, The equations $3 x^{2}-2=y^{2}$ and $8 x^{2}-7=z^{2}$. Quart. J. Math. Oxford Ser. (2) 20 (1969), 129-137.

[3] A. BAKER, G. WÜstholz, Logarithmic forms and group varieties. J. Reine Angew. Math. 442 (1993), 19-62.

[4] M. A. Bennett, On the number of solutions of simultaneous Pell equations. J. Reine Angew. Math. 498 (1998), 173-199.

[5] Yu. Bilu, G. HANROT, Solving Thue equations of high degree. J. Number Theory, 60 (1996), 373-392.

[6] J. H. Chen, P. M. Voutier, Complete solution of the Diophantine equation $X^{2}+1=d Y^{4}$ and a related family of Thue equations. J. Number Theory 62 (1996), 273-292.

[7] A. Dujella, B. JadriJević, A parametric family of quartic Thue equations. Acta Arith. 101 (2002), 159-170.

[8] A. Dujella, B. JadriJević, A family of quartic Thue inequalities. To appear in Acta Arith. 
[9] A. Dujella, A. Pethö, A generalization of a theorem of Baker and Davenport. Quart. J. Math. Oxford Ser. (2) 49 (1998), 291-306.

[10] C. Heuberger, A. Pethö, R. F. Tichy, Complete solution of parametrized Thue equations. Acta Math. Inform. Univ. Ostraviensis 6 (1998), 93-113.

[11] B. JADRIJEvić, A two-parametric family of quartic Thue equations. PhD thesis, University of Zagreb, 2001. (in Croatian)

[12] B. JADRIJEVIĆ, A system of Pellian equations and related two-parametric family of quartic Thue equations. Rocky Mountain J. Math. 35 no. 2 (2005), 547-571.

[13] G. Letth, A. Peтнő, Complete solution of a family of quartic Thue equations. Abh. Math. Sem. Univ. Hamburg 65 (1995), 365-383.

[14] W. Ljunggren, Über die Gleichung $x^{4}-D y^{2}=1$. Arch. Math. Naturvid. 45 (1942), 1-12.

[15] M. Mignotte, A. Pethö, R. Roth, Complete solutions of quartic Thue and index form equations. Math. Comp. 65 (1996), 341-354.

[16] A. Ретно̋, Complete solutions to families of quartic Thue equations. Math. Comp. 57 (1991), 777-798.

[17] A. Pethö, R. Schulenberg, Effectives Lösen von Thue Gleichungen. Publ. Math. Debrecen 34 (1987), 189-196.

[18] A. Ретнӧ, R. T. ТІсну, On two-parametric quartic families of Diophantine problems. J. Symbolic Comput. 26 (1998), 151-171.

[19] E. Thомаs, Complete solutions to a family of cubic Diophantine equations. J. Number Theory 34 (1990), 235-250.

[20] A. THUE, Über Annäherungswerte algebraischer Zahlen. J. Reine Angew. Math. 135 (1909), 284-305.

[21] A. ToGBÉ, On the solutions of a family of quartic Thue equations. Math. Comp. 69 (2000), 839-849.

[22] N. Tzanakis, Explicit solution of a class of quartic Thue equations. Acta Arith. 64 (1993), 271-283.

[23] N. Tzanakis, B. M. M. DE WEger, On the practical solution of the Thue equation. J. Number Theory 31 (1989), 99-132.

[24] I. WAKABAYASHI, On a family of quartic Thue inequalities. J. Number Theory 66 (1997), 70-84.

[25] I. WAKABAYASHI, On a family of quartic Thue inequalities, II. J. Number Theory 80 (2000), 60-88.

[26] P. G. WALSH, A note a theorem of Ljunggren and the Diophantine equations $x^{2}-k x y^{2}+y^{4}=$ 1, 4. Arch Math. 73, No.2, (1999), 119-125.

Borka JADRIJEVIĆ

FESB, University of Split

R. Boškovića bb

21000 Split, Croatia

E-mail : borka@fesb.hr 